\title{
KM3NeT/ARCA sensitivity to a diffuse flux of cosmic neutrinos
}

\section{Stransky ${ }^{* a}$, R. Coniglione ${ }^{b}$ and L. A. Fusco ${ }^{c, d}$ for the KM3NeT Collaboration}

${ }^{a}$ Friedrich-Alexander-Universität Erlangen-Nürnberg,

Erlangen Centre for Astroparticle Physics, Erwin-Rommel-Str. 1, 91058 Erlangen, Germany.

Email:dominik. stranskyefau.de

${ }^{b}$ INFN-LNS, via S. Sofia 62, 95123 Catania, Italy.

Email:coniglionedlns.infn.it

${ }^{c}$ Dipartimento di Fisica e Astronomia dell'Università di Bologna, Viale Berti-Pichat 6/2, 40127, Bologna, Italy.

d INFN - Sezione di Bologna, Viale Berti-Pichat 6/2, 40127, Bologna, Italy.

E-mail: Ifusco@bo.infn.it

\begin{abstract}
$\mathrm{KM} 3 \mathrm{NeT}$ is a future research infrastructure hosting the next-generation underwater neutrino observatory in the Mediterranean Sea. Within KM3NeT, the ARCA detector will be devoted to the observation of high-energy cosmic neutrinos both in diffuse and point-source mode. A major objective of KM3NeT/ARCA is to perform all-flavour neutrino astronomy.

The observation of cosmic neutrinos has been reported by the IceCube collaboration in high energy analyses, exceeding significantly the expectations from atmospheric backgrounds. This observation is compatible with an isotropic cosmic flux, equally distributed in the three neutrino flavours.

The sensitivity of the planned ARCA detector, with an instrumented volume of about one Gton, to a diffuse neutrino flux has been investigated in all-flavour analyses and the results will be reported in this contribution. In particular, KM3NeT/ARCA will be able to detect an IceCube-like neutrino flux within less than one year of operation with the complete detector.
\end{abstract}

The 34th International Cosmic Ray Conference,

30 July-6 August, 2015

The Hague, The Netherlands

\footnotetext{
${ }^{*}$ Speaker.
} 


\section{Introduction}

KM3NeT [1] is the next-generation neutrino telescope being built in the Mediterranean Sea. A three-dimensional array of several thousand digital optical modules (DOMs) [2], each housing 31 3-inch photomultiplier tubes (PMTs), will constitute the detector. DOMs will be distributed along vertical strings [3] anchored to the sea-bed, each holding 18 of them at a spacing of 36 m. 115 strings arranged together will constitute a detector building block. Prototypes for the KM3NeT detector are already being tested under the sea and recent achievements are reported in Refs. [4, 5, 6].

The discovery of a diffuse cosmic neutrino flux by the IceCube collaboration $[7,8]$ has had a high impact in the field of neutrino astronomy. Knowing that high-energy cosmic neutrinos exist, the focus for future neutrino telescopes like KM3NeT lies in detecting the sources of such neutrinos. In its final state, KM3NeT will consist of 6 detector blocks comprising an instrumented volume of about $3 \mathrm{~km}^{3}$. As an intermediate construction step the ARCA detector is planned - two detector building blocks with a $1 \mathrm{~km}^{3}$ instrumented volume [9]. One of the goals of the ARCA telescope, besides finding galactic neutrino sources [10], is to detect the diffuse cosmic neutrino flux and confirm IceCube's results from a complementary field of view. Comprehensive analyses towards this goal have been performed and will be described in these proceedings.

The diffuse, isotropic cosmic neutrino flux (per flavour) assumed for this analysis is the IceCube best fit value reported in Ref. [7], with a cutoff at $3 \mathrm{PeV}$ added to account for the lack of events at the Glashow resonance:

$$
E_{v}^{2} \frac{d \Phi\left(E_{V}\right)}{d E_{v}}=1.2 \cdot 10^{-8} e^{-E_{v} / 3 \mathrm{PeV}} \mathrm{GeV} \mathrm{cm}^{-2} \mathrm{~s}^{-1} \mathrm{sr}^{-1}
$$

Moreover, in a recent IceCube analysis [11] with a lower energy threshold (about $1 \mathrm{TeV}$ ) a consistent flux was measured but with a different slope (again, a $3 \mathrm{PeV}$ cutoff is added):

$$
\frac{d \Phi\left(E_{v}\right)}{d E_{v}}=2.06 \cdot 10^{-18}\left(E_{V} / 10^{5} \mathrm{GeV}\right)^{-2.46} e^{-E_{V} / 3 \mathrm{PeV}} \mathrm{GeV}^{-1} \mathrm{~cm}^{-2} \mathrm{~s}^{-1} \mathrm{sr}^{-1}
$$

The description of analysis results will focus on those for the flux of Eq. 1.1, but are similar for the flux of Eq. 1.2.

The major background for neutrino telescopes is given by atmospheric muons and neutrinos originating from the interactions of cosmic rays with the atmosphere. While atmospheric muons only come from above and can be topologically distinguished from neutrino-induced events, atmospheric neutrinos can only be statistically distinguished from their astrophysical counterparts. The present analyses uses the atmospheric neutrino flux models from Honda et al. [12] and Enberg et al. [13] that describe the conventional and prompt components, respectively. Following Ref. [14] a correction due to the presence of the knee in the cosmic ray spectrum was applied. Figure 1 (left) reports the assumed atmospheric neutrino fluxes compared to the IceCube fits above.

The signal and background neutrino fluxes can be separated due to their different energy spectra. In this contribution, a brief overview of the Monte Carlo simulation chain and of the reconstruction algorithms is given in Section 2. Then the sensitivity analyses and their results are presented in Section 3. 

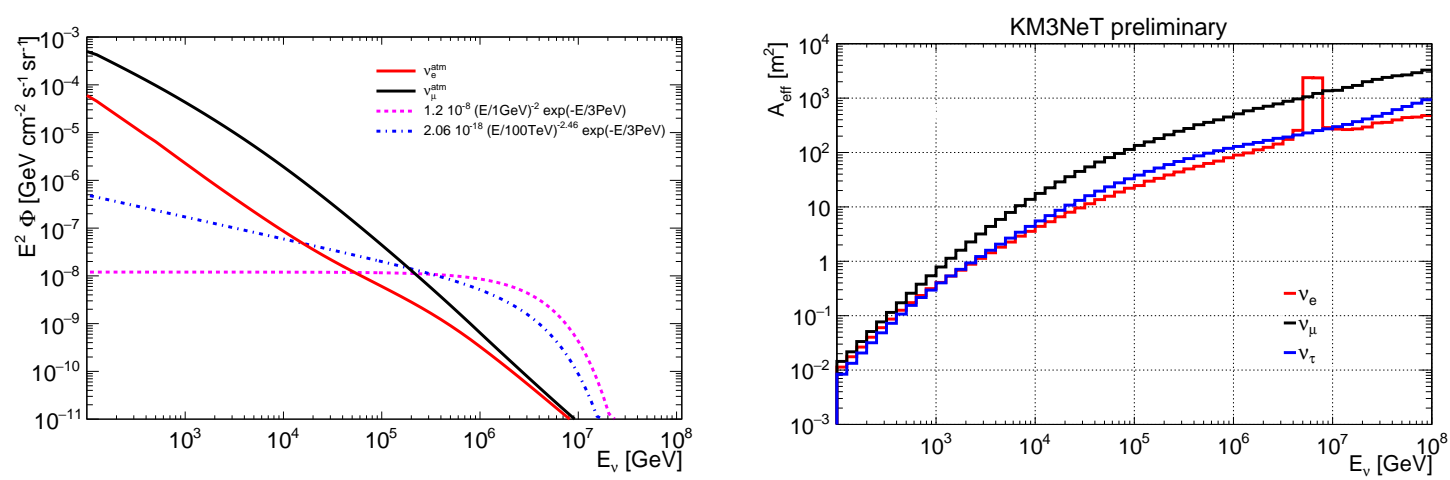

Figure 1: Left panel: signal and background neutrino fluxes as a function of the neutrino energy. Right panel: All-sky averaged neutrino effective neutrino areas (averaged over $v$ and $\bar{v}$ ) at trigger level as a function of the neutrino energy.

\section{Simulation and reconstruction}

Monte Carlo simulations have been performed covering all the physics processes relevant for the ARCA experiment: cascades produced by $v_{\mu}, v_{e}$, and $v_{\tau}$ neutral current (NC) and charged current (CC) events interacting in the vicinity of the detector [15], and individual through-going muons from $v_{\mu} \mathrm{CC}$ events up to many kilometres away [16].

Neutrino events from signal and background were simulated over the full sky and in the energy range from $10^{2}$ to $10^{8} \mathrm{GeV}$ with a live-time of over $10^{4}$ years above $10^{5} \mathrm{GeV}$. The generated events were weighted to reproduce the diffuse neutrino spectra and the atmospheric neutrino spectra reported in the left panel of Fig. 1.

The effective neutrino areas at trigger level are reported as a function of the neutrino energy for the three neutrino flavours in the right panel of Fig. 1. The effective area for $v_{\mu}$ events is much larger due to the long range of the outgoing muon from $v_{\mu} \mathrm{CC}$ interactions.

Atmospheric muons were generated with the fast MUPAGE code [17], which provides a parameterisation of the underwater flux of atmospheric muons, including multi-muon events (muon bundles). Two samples of atmospheric muon events were generated: the first for $E_{b}>10 \mathrm{TeV}$, where $E_{b}$ is the sum of the energies of all single muons in the bundle, with a corresponding livetime of about 3 months; and a second sample of events with $E_{b}>50 \mathrm{TeV}$, with 3 years of live-time. CORSIKA [18] has been used to estimate the 'self-veto' effect of atmospheric neutrinos and muons arriving in coincidence [19].

The track direction and energy reconstruction algorithms developed in the context of KM3NeT/ARCA are described in Ref. [16]. Three different shower reconstruction strategies are available [15], providing an extremely good angular and energy resolution. Besides reconstructing physical parameters like energy and direction, all of them provide different quality parameters to improve the selection of a high-purity sample.

\section{ARCA sensitivity to a diffuse flux}

Two analyses have been developed to search for a diffuse neutrino flux with ARCA. The 'track' analysis searches for through-going muon track events induced mainly by $v_{\mu} \mathrm{CC}$ interactions and 

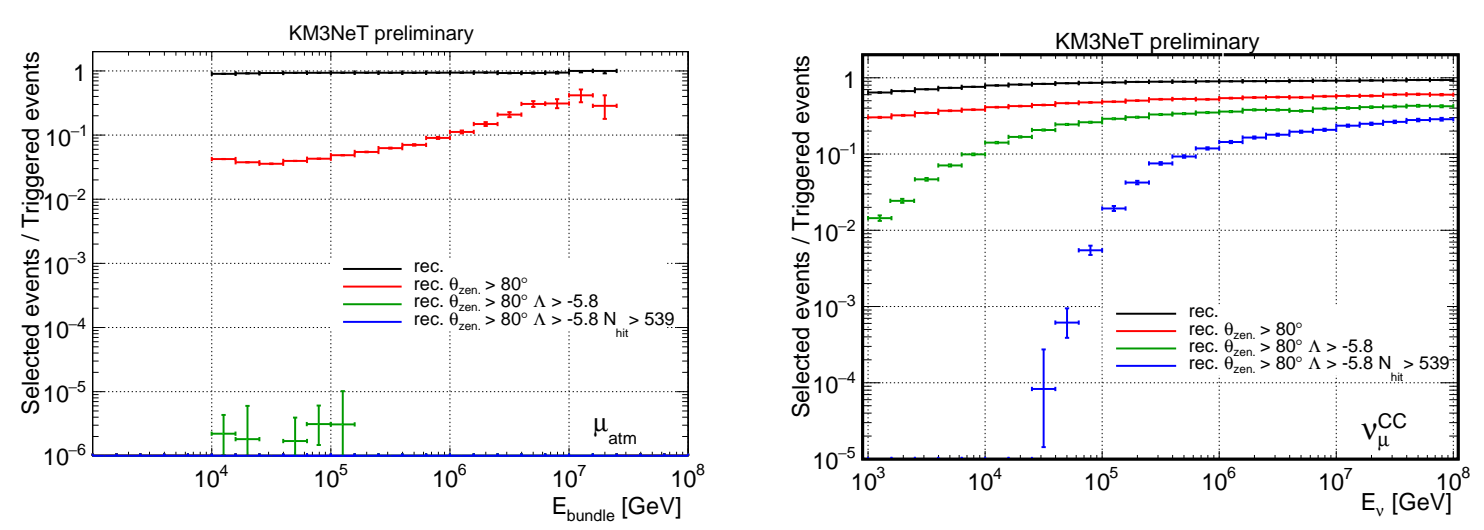

Figure 2: Ratio between the number of selected events and triggered events (Fig. 1 right) at each step of the track analysis (see text) for atmospheric muons (left panel) and $v_{\mu} \mathrm{CC}$ neutrinos (right panel) as a function of the neutrino energy.

by $v_{\tau} \mathrm{CC}$ events, if the secondary tau decays into a muon. The 'shower' analysis identifies allflavour neutrino interactions in the $\mathrm{NC}$ channel and the $\mathrm{CC}$ interactions of $v_{e}$ and $v_{\tau}$, which produce electromagnetic and hadronic showers at the interaction vertex. Both analyses have been optimised using the 'model discovery potential' (MDP) method for a $5 \sigma$ discovery of the spectrum given in Eq. 1.1 after one year's observation, by minimising the flux constant required to give a $50 \%$ chance of discovery. Each is described separately in their individual sections below, before their combined sensitivity to a flavour-uniform diffuse flux is calculated in Sec. 3.3.

\subsection{Track channel}

The first step in the event selection for the track channel analysis is a cut on the reconstructed track direction to get rid of most of the atmospheric muons. After optimisation a cut to events with zenith angle $\theta_{\mathrm{zen} .}^{\text {rec }}>80^{\circ}$ was chosen.

As shown in Fig. 2 (left), where the ratio of the selected events to the triggered events is reported at each step of the analysis for atmospheric muons, this cut does not reject all the atmospheric muons: a significant fraction of these events is wrongly reconstructed as up-going. To reject the background due to mis-reconstructed atmospheric muons and to atmospheric neutrinos the MDP minimisation is performed selecting well-reconstructed tracks and removing less energetic events mainly due to atmospheric neutrinos. The two reconstruction parameters correlated with the reconstruction quality and the neutrino energy are the $\Lambda$ and $N_{\text {hit }}$ parameters, described in Ref. [16]. Cuts of $\Lambda>-5.8$ and $N_{\text {hit }}>539$ proved optimal. Fig. 2 reports the ratio between selected events after these cuts and triggered events for atmospheric muons (left panel) and $v_{\mu}$ CC events (right panel). The angular and energy resolution for the final event selection is about $0.2^{\circ}$ and about 0.27 in the logarithm of the energy, respectively.

In one year of observation time, the final selected event sample contains 5.4 events from background and 7.6 events from the diffuse neutrino flux. With these event rates, the $5 \sigma$ discovery flux is approximately twice the assumed flux of Eq. (1.1). The sensitivity can be increased by using maximum likelihood methods on the simulated $N_{\text {hit }}$ distribution, rather than using a simple cut. This has been performed as described in [10], on a sample of preselected neutrino events with 
$\theta_{\mathrm{zen} .}^{\mathrm{rec}}>80^{\circ}$ and $\Lambda>-5.8$. The likelihood ratio function that has been maximised is given by:

$$
L R=\sum_{i=1}^{n} \log \frac{\frac{n_{\mathrm{sig}}}{n} \cdot P_{\mathrm{sig}}\left(N_{h i t}^{i}\right)+\left(1-\frac{n_{\mathrm{sig}}}{n}\right) \cdot P_{\mathrm{back}}\left(N_{h i t}^{i}\right)}{P_{\text {back }}\left(N_{h i t}^{i}\right)},
$$

where $n_{\text {sig }}$ is the number of signal events, $n=n_{\text {sig }}+n_{\text {back }}$ the total number of events, $n_{\text {back }}$ the number of background events and $P_{\text {sig }}$ and $P_{\text {back }}$ the probability distribution functions (PDF) for a signal or background to be reconstructed with a final selected number of hits $N_{h i t}^{i}$. This function is maximised with respect to the number of signal events $n_{\text {sig. }}$. The resulting significance is reported in Fig. 4 as a function of the number of observation years and is about $4 \sigma$ after one year with a $50 \%$ chance of detection. The same analysis has been also performed to test the IceCube flux of Eq. (1.2) and the results slightly improve.

\subsection{Shower channel}

An event selection procedure based on three consecutive cuts has been used in the shower analysis to isolate signal from background events. At the first selection level, only contained events are kept. Since most of the atmospheric muons enter the detector from above, their pseudo-vertices are reconstructed in the upper part of the detector. A large part of the background is then rejected by selecting on the vertical distance, $\mathrm{z}$, and on the radial distance, $\mathrm{R}$, of the reconstructed vertices with respect to the detector centre. The applied selection cut is $\mathrm{R}<500 \mathrm{~m}$ and $\mathrm{z}<200 \mathrm{~m}$. This cut reduces the detector geometrical volume by about $20 \%$.

A further rejection of atmospheric muons via an energy-related cut is obtained, cutting on the total time over threshold (ToT) of hits selected by the shower reconstruction algorithm. The ToT is measured for each PMT and is a measure of the collected charge [2]. A crude choice for this cut is made at $12 \mu \mathrm{s}$, rejecting a large number of low-energy atmospheric muons and also a large part of the atmospheric neutrino background, dominant at lower energies.

The final step to isolate the signal from the remaining background uses a machine-learning algorithm based on the boosted decision tree (BDT) from the ROOT TMVA package [20]. As input for the BDT training, several quality parameters from the available shower and track reconstruction algorithms are used. The BDT is then trained to discriminate tracks from showers using simulated atmospheric muons and $v_{e} \mathrm{CC}$ events as training samples. The output of the BDT, $\rho$, is used together with the reconstructed shower energy $E_{\text {rec }}$ to separate the signal from both the remaining atmospheric neutrino and muon backgrounds. The optimal cuts resulting from the MDP minimisation were found to be $\rho>0.50$ and $E_{\text {rec }}>10^{4.7} \mathrm{GeV}$, with final event rates of 16.1 for signal and 8.4 for the background in one year operation of ARCA. The mean angular and energy resolutions for these events are roughly $2^{\circ}$ and $10 \%$, respectively. In Fig. 3 the ratio of the selected to triggered events is reported at each selection step.

With these event rates in the shower channel a $5 \sigma$ discovery of the nominal IceCube flux of Eq. (1.1) can be achieved after 1.3 years of ARCA with $50 \%$ probability. A further improvement of this result is reached by applying the maximum likelihood method described in Section 3.1. The signal and background PDFs (Eq. 3.1) are two-dimensional functions of the reconstructed shower energy, $E_{\text {rec }}$, and the BDT output, $\rho$. The resulting significance as a function of the observation years is reported in Fig. 4. The same analysis has also been performed to test the IceCube flux of Eq. (1.2) and the results slightly improve. 

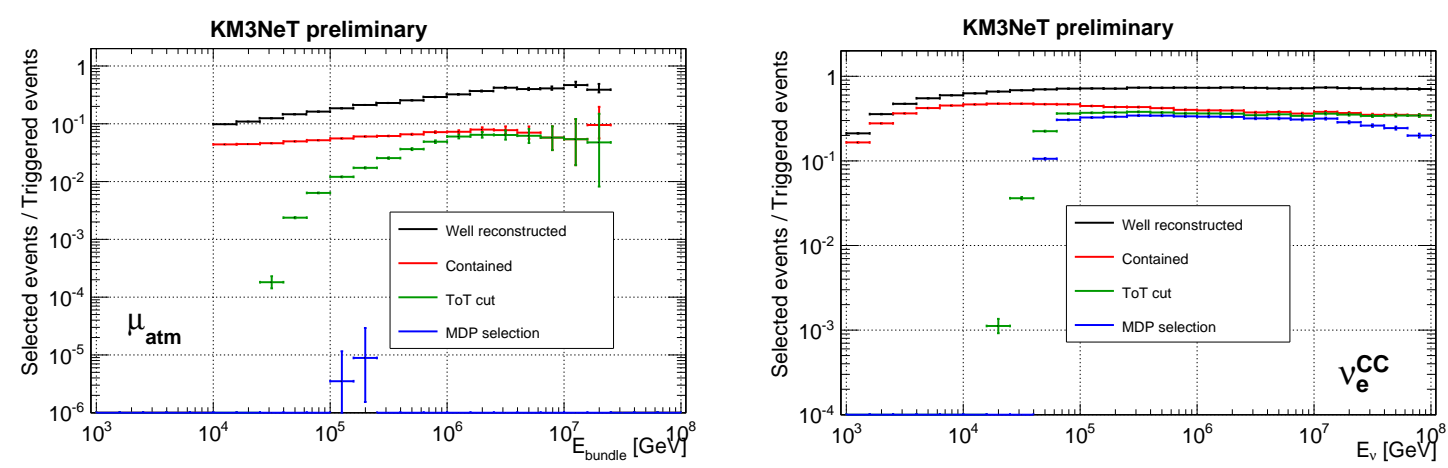

Figure 3: Ratio between the number of selected events and triggered events (Fig. 1 right) at each step of the shower analysis (see text) for atmospheric muon events (left panel) and $v_{e} \mathrm{CC}$ events (right panel) as a function of the neutrino energy.

For the shower channel a second alternative analysis has been developed based on fitting four separate components: standard and prompt atmospheric neutrino fluxes, misidentified atmospheric muons, and a cosmic diffuse flux. This analysis does not use a preselected event sample and takes into account most of the information from the signal events; only loose cuts rejecting low-quality events are applied. These events are used for a two-step-training of a machine-learning algorithm based on the BDT. In the first step the BDT is trained to discriminate track-like events versus cascade-like events using some of the parameters provided by the reconstruction codes. The BDT assigns at each event a $\rho$ value according to its probability of being shower or cascade. The BDT output is then used, together with other parameters, as input for the training of the second BDT to discriminate signal-like events versus background-like events. The output of the second BDT is then the variable for a fitting method based on a Poissonian maximum likelihood approach. The normalisations of all four sources of events are fitted, keeping the spectral indices fixed. With a possible $5 \sigma$ discovery after roughly 1 year of ARCA a similar result is achieved compared to the other shower analysis, since the loss from fitting four components rather than one is offset by the gain in sensitivity from using all available reconstruction output.

\subsection{Combined track and shower analysis}

As both the track and shower analyses have a strong bias in efficiency towards one particular event topology, a further analysis strategy has been developed to incorporate the results from both the searches. The two analyses are indeed almost independent, as the overlap between the final event samples is limited to less then $2 \%$ of the surviving events (mostly $v_{\mu} \mathrm{CC}$ events with energy above $10^{4.5} \mathrm{GeV}$ with the muon crossing the detector).

In order to develop an all-sky analysis with tracks and showers, the two hemispheres are treated separately. For downward-going events, where the atmospheric background is extremely high, only contained shower events are considered since muon-induced neutrinos would be completely covered by atmospheric muons. The selection for these events is completely analogous to that of the usual shower analysis.

For upgoing events, preliminary cuts are used to reject part of the atmospheric muons that are wrongly reconstructed as upgoing, then a BDT multivariate technique is used to provide a further rejection of these events. This BDT uses parameters coming from both the track and shower 
reconstruction algorithms and is optimised to reject atmospheric muons. Its output is used together with the shower energy estimation in the same maximum likelihood approach reported in 3.1.

The result of this combined analysis is equivalent to the combination of the two individual results from the track and shower analyses. A $4.8 \sigma$ significance is obtained in 0.5 years of observation time.

\subsection{Systematic uncertainties}

Comprehensive studies on the possible systematic uncertainties have been performed for these analyses. Uncertainties on the DOMs orientation of up to $9^{\circ}$ in the azimuthal rotation angle, $\pm 10 \%$ in the total PMT collection efficiency, $\pm 10 \%$ in the absorption and scattering properties of water, and uncertainties in the atmospheric neutrino flux, were taken into account. The magnitudes of each systematic were chosen to reflect current maximal uncertainties in these values - when ARCA is built, far better calibration information will be available.

For the assumed detector and water uncertainties no significant impact $(<10 \%$ in the shower channel and $<6 \%$ in the track channel) on the discovery potential was found.

The assumed uncertainty in the conventional atmospheric neutrino flux was of $\pm 25 \%$ in the normalisation of the Honda et al. flux model adopted. While this has only a minor effect on the sensitivity in the shower channel, it is the major uncertainty for the track channel with a result of $\pm 10 \%$ in the discovery flux. On the contrary the largest effect in the shower channel is given by the uncertainty of the prompt atmospheric neutrino flux, which is chosen according to the maximum and minimum predicted by the Enberg et al. model [13]. The effect on the sensitivity can be amounted to roughly $\pm 30 \%$. These uncertainties are reported as bands into the significance as a function of time shown for each channel in Fig. 4.

\section{Conclusion}

The IceCube discovery of an extraterrestrial high-energy neutrino flux has led the KM3NeT collaboration to plan an intermediate step in the construction of a high-energy neutrino telescope in the Mediterranean Sea. This detector, called ARCA, consists of two detector blocks of 115 strings, each with a total instrumented volume of about $1 \mathrm{~km}^{3}$. In this paper a preliminary study to evaluate the discovery potential of the future ARCA neutrino telescope to the discovered IceCube flux has been presented.

The all-flavour analysis based on a complete Monte Carlo simulation predicts that the time of operation of the ARCA detector required to detect the IceCube flux at a significance level of $5 \sigma$ is of about 0.5 years. If this prediction is confirmed by the observation a further understanding on the origin of this flux can be achieved thanks to the complementary field of view and the high angular and energy resolution of the ARCA detector [15].

\section{References}

[1] www.km3net.org.

[2] R. Bruijin et al., for the KM3NeT Collaboration, proceedings of this conference ID 1157.

[3] P. Kooijman et al., for the KM3NeT Collaboration, proceedings of this conference ID 1173. 


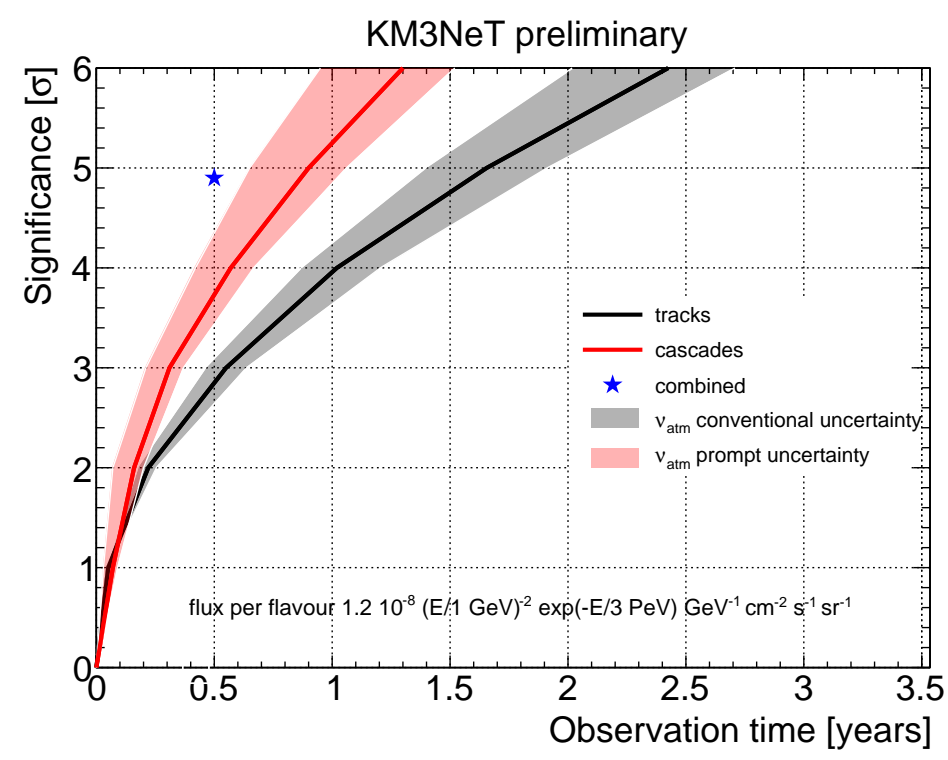

Figure 4: Significance as a function of observation time for the detection of a diffuse flux of neutrinos corresponding to the signal reported by IceCube, in the up-going muon channel (black), the cascade channel (red) and the combined analysis (blue star). The bands indicate the maximum values of uncertainties.

[4] S. Biagi et al., for the KM3NeT Collaboration, proceedings of this conference ID 1164.

[5] A. Creusot for the KM3NeT collaboration, proceedings of this ID 1154.

[6] S. Adrian Martinez et al., Eur. Phys. J. C 743056 (2014).

[7] M. G. Aartsen et al. (IceCube Coll.), 2013 Science 3421242856.

[8] M. G. Aartsen et al. (IceCube Coll.), Phys. Rev. Lett. 113101101 (2014).

[9] P. Piattelli et al., for the KM3NeT Collaboration, proceedings of this conference ID 1158.

[10] A. Trovato et al., for the KM3NeT Collaboration, proceedings of this conference ID 1113.

[11] M. G. Artsen et al., Phys. Rev. D 91022001 (2015).

[12] M. Honda et al., Phys. Rev. D 75043006 (2007).

[13] R. Enberg et al., Phys. Rev. D 78043005 (2008).

[14] M. G. Artsen et al., Phys. Rev. D 89062007 (2014).

[15] D. Stransky et al. for the KM3NeT Collaboration, proceedings of this conference ID 1108.

[16] A. Trovato et al. for the KM3NeT Collaboration, proceedings of this conference ID 1114.

[17] Y. Becherini et al., Astropart. Phys. 25 (2006) 1.

[18] D. Heck et al., (https://www.ikp.kit.edu/corsika/), Wissenschaftliche Berichte, Forschungszentrum Karlsruhe FZKA 6019 (1998).

[19] T. Heid (for the KM3NeT Collaboration), proceedings of this conference ID 1067.

[20] A. Hoecker, P. Speckmayer, J. Stelzer, J. Therhaag, E. von Toerne, and H. Voss, TMVA - Toolkit for Multivariate Data Analysis, PoS ACAT 040 (2007), arXiv:physics/0703039. 